\title{
Informational Content Components \\ to Ensure Problem-Conflict Stability Exercises \\ and Training Plans, Training \\ of Operators Ergotechnical Simulator Radio \\ Electronic Facilities
}

\author{
Andrey N. Potapov* \\ Military Education and Research Centre of Military-Air Forces \\ «Military-Air Academy \\ Named After Professor N.E. Zhukovsky and Yu.A. Gagarin» \\ 54a Starykh Bolshevikov Str., Voronezh, 394064, Russia
}

Received 25.02.2016, received in revised form 13.07.2016, accepted 24.09.2016

Consider the problem of creating conflict-resistant automated control system simulator preparation of operators of ergotechnical radio electronic facilities, and information security components of the content of the issue of conflict-stability exercises and training plans for training of operators ergotechnical simulator radio electronic facilities.

Keywords: operator, radio-electronic means, training exercises and plans, simulator training, automatic control system.

Citation: Potapov A.N. Informational content components to ensure problem-conflict stability exercises and training plans, training of operators ergotechnical simulator radio electronic facilities, J. Sib. Fed. Univ. Eng. technol., 2016, 9(8), 1217-1228. DOI: 10.17516/1999-494X-2016-9-8-1217-1228.

(C) Siberian Federal University. All rights reserved

* Corresponding author E-mail address: anpotapov36@yandex.ru 


\title{
Информационные компоненты содержания \\ вопроса обеспечения конфликтоустойчивости \\ учебных упражнений и планов тренажной подготовки \\ операторов эрготехнических радиоэлектронных средств
}

\author{
А.Н. Потапов \\ Военный учебно-научный цуентр Военно-воздушных сил \\ «Военно-воздушная академия \\ имени профессора Н.Е. Жуковского и Ю.А. Гагарина» \\ Россия, 394064, Воронеж, ул. Старых Большевиков, 54 а
}

Рассмотрена проблема создания конфликтно-устойчивой автоматизированной системь управления тренажной подготовкой операторов эрготехнических радиоэлектронньх средств, а также информационные компоненты содержания вопроса обеспечения конфликтоустойчивости учебных упражнений и планов тренажной подготовки операторов эрготехнических радиоэлектронных средств.

Ключевые слова: оператор, радиоэлектронное средство, учебные упражнения и планы, тренажная подготовка, автоматизированная система управления.

\section{Введение}

Современная система тренажной подготовки (ТП) операторов эрготехнических радиоэлектронных средств (РЭС) подвержена конфликтам, которые являются объективной действительностью как в организационно-технических, так и в психолого-физиологических аспектах. Причем организационно-техническая и психолого-физиологическая стороны конфликтов ТП взаимосвязаны. Это необходимо учитывать при определении мер по их защите от возможных конфликтов. Уровень ТП операторов по применению РЭС существенно влияет на эффективность выполнения боевых задач. Поэтому для прогнозирования эффективности выполнения частями и подразделениями мероприятий с привлечением РЭС необходимо учитывать реальный уровень подготовленности операторов. Наложение ряда экономических и организационнотехнических проблем организации ТП не только не позволяет обеспечить реализацию курсов ТП операторов в полном объеме, но и способствует возникновению конфликта боевого применения РЭС, который является объективной реальностью, независимо от принятых представлений по планированию боевых задач. Сущность конфликта боевого применения РЭС состоит в наличии противоречий между прогнозируемым и действительно необходимым количеством сил и средств, обеспечивающих гарантированную эффективность.

\section{Содержание вопроса по созданию конфликтно-устойчивой}

автоматизированной системы управления тренажной подготовкой операторов эрготехнических радиоэлектронных средств

В преломлении традиционных и современных принципов организации традиционного обучения для системы ТП операторов РЭС объектом управления является оператор. А в каче- 
стве субъекта управления выступают инструктор и непосредственно система ТП, так как она не заменяет, а дополняет инструктора. Системы управления ТП можно разделить на две группы: селективные (традиционные) (рис. 1) и интеллектуальные (автоматизированные) (рис. 2) [1].

В селективных системах управление ТП (определение содержания и последовательности упражнений $\Omega_{S}$, а также количества тренировок $N_{m p}$ ) осуществляется в соответствии с курсами ТП. Это, как было определено ранее, вполне соответствует современной ТП операторов РЭС. При этом каждый оператор проходит один и тот же путь ТП, т.е. отсутствует адаптация $N_{m p}$ к каждому конкретному обучаемому. Как правило, селекция заключается в выборе перечня упражнений и количества тренировок в соответствии с периодом подготовки оператора. При этом ТП обычно проводится на штатной технике (ШТ) с ограниченными возможностями по реализации стандартных операций $D$ и имитации типовых радиочастотных (РЧ) РЧ-условий $\Delta$

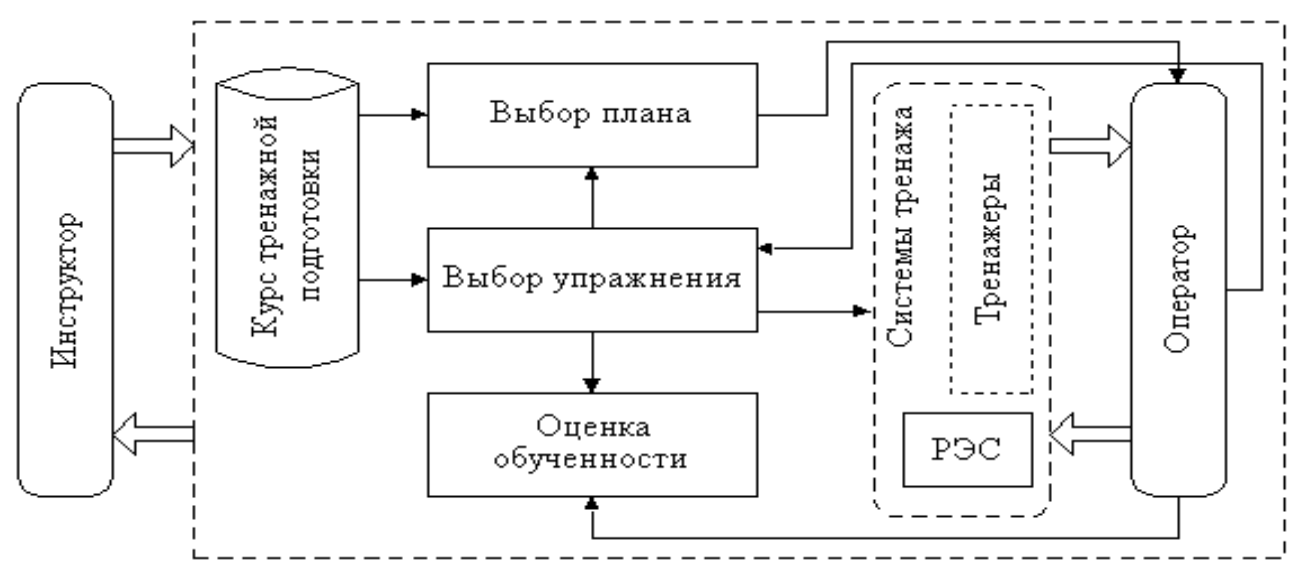

Рис. 1. Селективная (традиционная) система управления тренажной подготовкой операторов радиоэлектронных средств

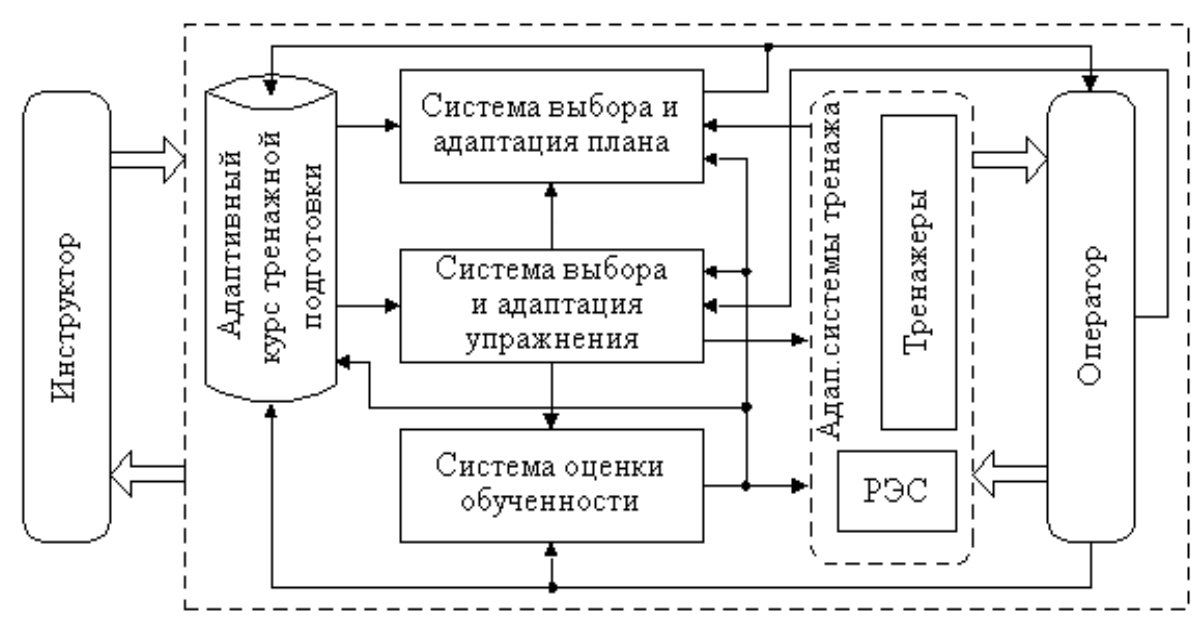

Рис. 2. Интеллектуальная (автоматизированная) система управления тренажной подготовкой операторов радиоэлектронных средств 
применения РЭС. Кроме того, из-за ограниченного эксплуатационного ресурса РЭС порой не представляется возможным проведение дополнительных тренировок в интересах достижения требуемого уровня подготовки операторов.

Современное состояние методологии, теории и практики автоматизации оценки операторской деятельности, построения экспертных систем и систем искусственного интеллекта позволяет развить селективную систему управления ТП до интеллектуального уровня [2] (рис. 2).

В интеллектуальной системе управления ТП в зависимости от ситуаций (комбинаторик различных ограничений) производится выбор упражнений и их содержания, планов и их наполнения, а также средств тренажа не только в зависимости от периода подготовки операторов, но и с учетом их индивидуальных особенностей (начального уровня обученности и скорости усвоения упражнений).

Помимо этого современная теория и практика отечественного [3] и зарубежного тренажеростроения и развитие вычислительной техники позволяют создавать недорогие по сравнению со ШТ тренажеры РЭС, которые в значительной степени расширяют возможности реализации стандартных операций $D$ и имитации типовых РЧ-условий применения РЭС. Это порой невозможно сделать в существующих образцах тренажных средств, что вызывает у самих операторов негативное отношение к ним. Кроме того, появились новые научные школы и направления, которые развивают основы тренажеростроения с позиции совершенствования теоретических основ адаптации тренажеров к состоянию оператора с выдачей ему рекомендаций методического и практического характера.

Интеллектуальная система управления ТП по результатам тренировок позволяет производить адаптацию самих курсов ТП, которые в настоящее время формируются на длительные сроки (5-10 лет) и не учитывают возможности уточнения упражнений и планов. Необходимость уточнения упражнений возникает при выявлении в процессе ТП более рациональных операций применения РЭС по типовым ситуациям, отражающим реальные способности операторов.

Однако, несмотря на несомненные преимущества автоматизированной (интеллектуальной) системы перед традиционной (селективной), ей свойственны следующие неразрешенные конфликты:

- конфликт применения РЭС, выраженный в противоречии между предписанными и альтернативными содержательными компонентами учебных упражнений, отражающих соответственно принятые и действительные РЧ-условия функционирования и операции применения РЭС;

- конфликт применения тренажеров, заключающийся в противоречии между оценками уровней навыков, получаемыми операторами РЭС при отработке единых учебных упражнений на тренажерах и ШТ, что приводит к формированию некорректных содержательных компонентов учебных планов, отражающих количество тренировок на средствах тренажа [4].

Эти конфликты с психолого-физиологической точки зрения способствуют формированию заведомо искаженных, а значит, ложных знаний (перцептивных образов) и навыков (умений выполнять целенаправленные мыслительные действия с апперцептивными и перцептивными образами), которые порой устранять сложнее, чем приобретать новые знания и навыки. В целом

$$
-1220-
$$


эти конфликты вредно влияют на полезность боевого применения РЭС, от которых, безусловно, необходима защита. Вопросы защиты систем обучения, в том числе и АС управления ТП, от этих, как, впрочем, и от других конфликтов в такой постановке, ранее не рассматривались. Защита подготовки военных операторов в основном была направлена на обеспечение: безопасности здоровья личного состава, скрытности тактических приемов боевого применения и режимов функционирования РЭС, конфиденциальности курсов боевой подготовки (КБП), организационно-методических указаний (ОМУ) и планов проведения войсковых учений. Конфликтоустойчивость автоматизированной системы управления (AC) управления ТП операторов, понимаемая в узком смысле, - способность к выявлению полезных навыков из смеси их с ошибочными навыками за счет селективных возможностей АС. При этом селективные возможности в традиционных системах управления ТП определяются в основном квалификацией инструкторов, которые в процессе отработки учебных упражнений операторами, обнаруживая отклонения их действий от предписанных действий, осуществляют методические приемы по устроению ошибочных действий и недоведению их до автоматизма, т.е. недопущению формирования ошибочных навыков.

С учетом тенденций развития методов имитационного моделирования и вычислительной техники появились реальные предпосылки замещения традиционных инструкторов электронными, которые выполняют в автоматизированном виде функции первых, что позволяет устранять субъективный фактор при защите ТП от ошибочных навыков. Поэтому конфликтоустойчивость АС управления ТП в узком смысле непосредственно зависит от корректности разработки электронных инструкторов. Кроме этого, селективные возможности АС управления ТП можно несколько расширить за счет автоматизации методов профессионального отбора на начальной стадии обучения и методов проверки профессиональной годности - в процессе учебной и боевой профессиональной деятельности операторов и т.п.

В настоящее время в теоретическом плане достаточно полно с позиций педагогики и психологии разработаны методологические основы, а именно теория оценки эрготехнических систем, сформированная профессором А.А. Безбоговым, теоретические основы оценки операторской деятельности, представленные в научных изданиях, В.С. Зайцева и Г.Н. Шибанова [5], которые напрямую не относятся к конфликтоустойчивости АС обучения, но позволяют обеспечить адаптивное управление курсами ТП операторов по оценкам их деятельности соответственно на тренажерах и на ШТ. При этом объектом управления является оператор, для которого в качестве входных параметров задаются виды и последовательности упражнений, их объемы и продолжительности, а в качестве выходных - оценки его деятельности. Оценки деятельности сравниваются с нормативами, и на основании анализа невязки между ними производится коррекция входных параметров оператора. В такой постановке можно считать, что автоматизация подготовки косвенно направлена на защиту в широком смысле от конфликта, вызванного отсутствием в КБП содержательных компонентов, отражающих в полной мере индивидуальные свойства обучаемых. Однако при этом не учитывается ограниченная адекватность имитационного моделирования в тренажерах, что приводит изначально к формированию некорректных оценок деятельности операторов, следовательно, его входных параметров, а значит, не достигается в полной мере положительный эффект защиты. 
Теоретические исследования по защите АС управления подготовкой операторов от возможных конфликтов применения ШТ и тренажеров практически не проводились. Хотя это научное направление актуально при постоянной модернизации как РЭС и тренажеров, так и РЧ-условий их функционирования. Основой защиты от этих конфликтов служит формирование операций боевого применения РЭС и определение количества тренировок, синтезируемых при решении задач выбора и распределения функциональных действий операторов и средств тренажа. Вопросы диагностики содержательных компонентов КБП операторов на этапе изменения РЧ-условий применения РЭС и тренажеров полностью выпадают из сферы деятельности лиц, принимающих решения по организации ТП. Стремление на основе принципа сквозного анализа конфликтов ТП наилучшим образом распределить содержательные компоненты КБП с использованием тренажеров так, чтобы удовлетворить реализуемость их полного решения, является важной и актуальной задачей. В интересах конфликтоустойчивости возникают проблемные вопросы в рамках автоматизации механизмов генерации содержательных компонентов учебных упражнений и учебных планов, которые обеспечат выполнение разнообразных переходов от одних РЧ-условий функционирования РЭС к другим с учетом ограниченной адекватности тренажеров.

В интересах защиты от конфликта применения РЭС предлагается дополнить АС управления ТП (рис. 3): системой формирования упражнений в соответствии с действительными РЧ-условиями функционирования РЭС; системой идентификации РЧ-условий взаимодействия РЭС со средой [6]. А для защиты от конфликта применения тренажеров можно включить в состав АС управления ТП систему оценки адекватности тренажеров по отношению к ШТ [7]. При этом конфликтоустойчивость АС управления ТП операторов понимается не в узком смыс-

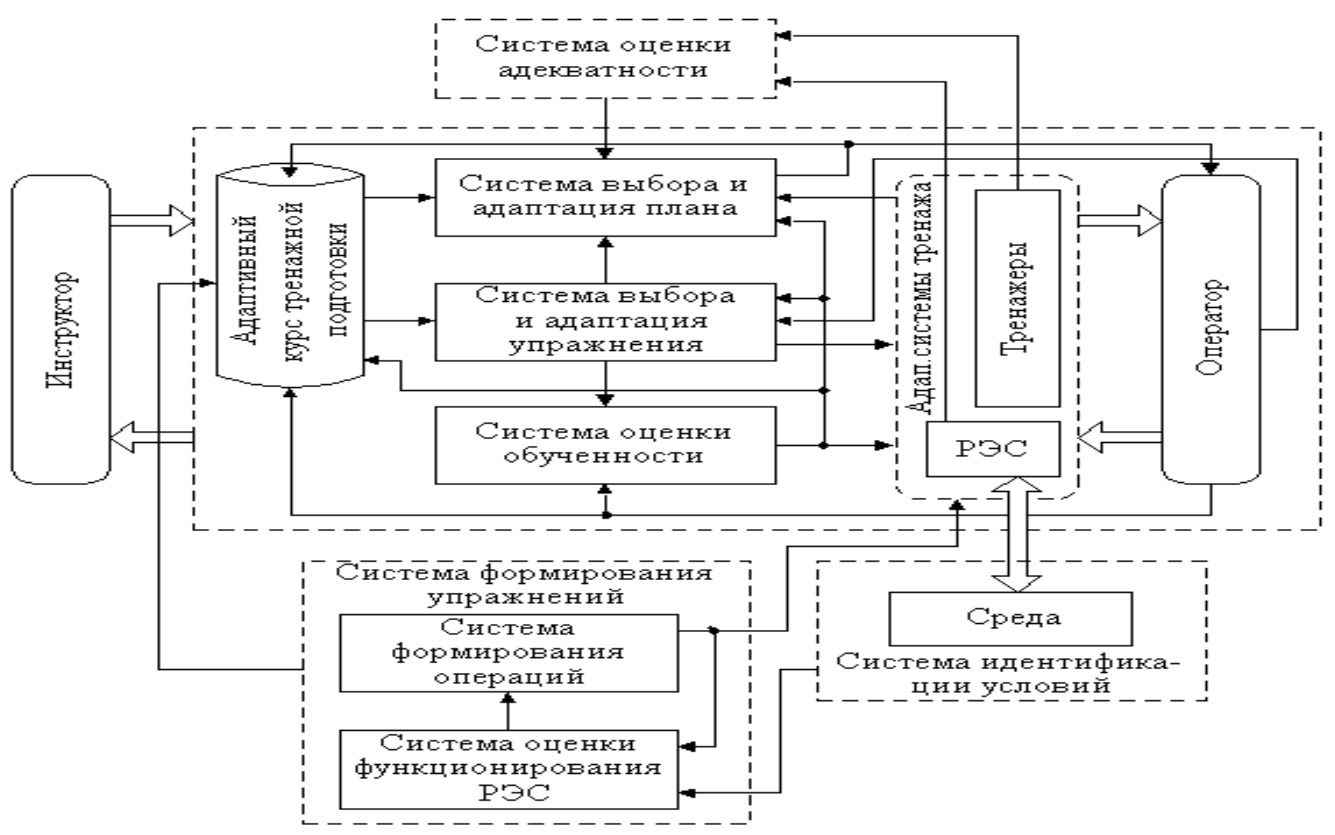

Рис. 3. Предлагаемая автоматизированная система управления тренажной подготовкой операторов радиоэлектронных средств 
ле - как способность к выявлению полезных навыков из смеси их с конфликтными, вредно влияющими на эффективность боевого применения РЭС за счет селективных возможностей $\mathrm{AC}$, а в широком - как способность к выявлению полезных содержательных компонентов учебных упражнений и учебных планов, определяющих КБП, в условиях конфликтов, вызванных изменяющимися РЧ-условиями функционирования РЭС и ограниченной адекватностью тренажеров.

\section{Информационный компонент содержания вопроса по защите содержательных компонентов учебных упражнений от конфликта применения эрготехнических радиоэлектронных средств}

Содержательными компонентами любого учебного упражнения, предназначенного для его отработки операторами РЭС, являются РЧ-условия функционирования и предписанные операции боевого применения РЭС. Предписанные операции в некоторых источниках схемы боевого применения, как правило, определяются эмпирически в ходе специальных исследовательских учений с привлечением экспертов, обладающих опытом боевой эксплуатации РЭС. Эпизодическая коррекция КБП осуществляется на основании ОМУ, формирующихся по результатам боевого применения РЭС на войсковых учениях, проведение которых в современных условиях затруднительно, что способствует моральному устареванию учебных упражнений как из-за внепланового усовершенствования самих РЭС, так и из-за изменения РЧ-условий их функционирования.

Кроме того, необходимо отметить следующее. Обычно специальные исследовательские и войсковые учения проводятся на специализированных полигонах со свойственными для них организационно-техническими проблемами, что, безусловно, способствует возникновению конфликта применения РЭС.

Известно, что в процессе боевого применения РЭС в соответствии с РЧ-условиями его функционирования оператор должен выбирать и осуществлять такую операцию $D=\left\{D_{r}\right\}$, которая позволила бы формировать реакцию $\operatorname{Re}(S)$ объекта в интересах достижения желаемого результата $P_{S}$. От корректности выбора, своевременности и точности выполнения операции $D$ зависит достижимость желаемого результата $P_{S}$. Условия функционирования РЭС, определяемые структурой окружающей среды и общностью РЧ-спектров ее элементов $Q_{j}$, могут оказывать существенное влияние на достижимость желаемого результата $P_{S}$. При этом в зависимости от сложившихся РЧ-условий операторский персонал производит реализацию регламентируемых, ранее отработанных им в ходе ТП операций $D_{S}^{\prime}$ по управлению РЭС.

В настоящее время из-за отсутствия инструментария, позволяющего адекватно адаптировать операции $D_{S}^{\prime}$ управления РЭС с учетом динамики изменения РЧ-условий его функционирования, регламентирующие операции по их управлению оказаться конфликтными [8], т.е. операции $D_{S}^{\prime}$ малоэффективны, а результат нежелательный, хотя в принципе РЭС вполне способен его получить.

Поэтому первой актуальной научной задачей является разработка метода формирования содержательного компонента учебных упражнений - операций, защищенных от конфликта применения РЭС, возникающего из-за изменения РЧ-условий, которые характеризуют текущие отношения в РЧ-спектре между РЭС и окружающей средой. 
Эта задача может быть решена на основании автоматизации процесса анализа РЧусловий функционирования РЭС и синтеза возможных ситуаций по его управлению в интересах обеспечения оперативной адаптации операций управления РЭС к изменяемым условиям их функционирования, которые должны отражаться в упражнениях ТП операторов как их содержательные компоненты. Для этого необходимо определить показатели и возможные взаимные отношения между операциями $D_{S}^{\prime}$ и $D_{Q}^{\prime}$ соответственно объекта $S$ и РЭС среды $Q$ и построить системную модель формирования этих операций, инвариантную к их целевому предназначению [4].

В современных условиях анализ РЧ-условий функционирования РЭС возможен на основании проведения радиомониторинга. Конечной целью радиомониторинга является диагностика и прогноз эффективности функционирования РЭС $S$. В настоящее время для автоматизации радиомониторинга широко используется вычислительная техника, где моделируются условия взаимодействия объектов радиомониторинга, а именно объектов $S$ и элементов среды $Q$. При этом для моделирования этих условий изначально необходимо производить идентификацию как объектов $S$, так и среды $Q$ с целью выбора их математических моделей. Однако из-за отсутствия единой формализованной классификации объектов $S$ и элементов среды $Q$, отражающей особенности целевого и функционального предназначения, а также принципов использования ими РЧ-спектра, имеются затруднения в автоматической конфигурации математических моделей объектов мониторинга, что снижает его оперативность. С учетом этого актуален поиск возможных вариантов единой формализованной классификации объектов радиомониторинга, которая стала бы основой для создания программного обеспечения автоматического управления конфигурацией математических моделей в АС радиомониторинга.

При общности РЧ-спектра, состоящего из основных и побочных каналов излучения и приема, мониторинг функционирования РЭС, как правило, базируется на выявлении между ними связанности типа «выход $S_{j}$ - вход $S_{k}$ », где $S_{j} \subset S, S_{k} \subset S, j, k=\overline{1, N}$. Под выходом $S_{j}$ понимается спектр излучений радиопередатчиков, а под входом $S_{k}$ - частотная область радиоприема. Однако помимо данного типа связанности существуют такие, как «выход $S_{j}-$ выход $S_{k}$ », «вход $S_{j}$ - вход $S_{k}$ ». Очевидно, что объекты $S_{j}$ и $S_{k}$ могут оказывать взаимное влияние друг на друга через комбинаторику этих типов связанности. Поэтому уместно детализировать взаимные системные отношения в структурно-параметрическом представлении для каждого типа связанности.

В настоящее время имеется ряд работ, посвященных вопросам синтеза схем применения РЭС на основании анализа информационных конфликтов [9], свойственных условиям их взаимодействия со средой. При этом информационный конфликт определяет меру взаимодействия РЭС и среды. На самом деле топология взаимодействия между РЭС и средой несколько шире, а именно их элементы дополнительно могут быть в отношениях сотрудничества, безразличия и независимости. Нет четкого системного описания условий взаимодействия РЭС и среды, а тем более ее топологии, что не позволяет оценивать конфликт, сотрудничество, безразличие и независимость между ними, а значит, корректно определять операции по способам его применения. А без идентификации РЧ-условий функционирования РЭС, являющихся сопутствующим признаком конфликта их применения, задача по защите от этого конфликта теряет свой смысл. 


\section{Информационный компонент содержания вопроса \\ по защите содержательных компонентов учебных планов от конфликта применения тренажеров}

Известно, что основой построения современных тренажеров служит имитационное моделирование процессов, свойственных ШТ. Однако любая модель - некоторое приближение к реальному объекту. От уровня формализации и учета различных факторов при создании моделей зависит степень ее приближения к реальному объекту, т.е. адекватность. Ввиду того что даже при современных возможностях создания имитационных моделей нельзя учесть все факторы, роль тренировок на ШТ остается значимой. Неадекватность тренажеров можно уменьшать до некоторого предельного уровня, но при этом их стоимость будет возрастать. Реальна такая ситуация, когда при достижении предельно возможного уровня неадекватности тренажера стоимость тренировок на тренажере может превысить стоимость тренировок на ШТ. А это нарушает саму идею создания тренажеров [2]. На основании этого сделаем следующий вывод: неадекватность тренажеров по отношению к ШТ не может быть полностью устранима. Решение задачи по полному устранению неадекватности тренажера возможно лишь при превращении его в ШТ. В таком тренажере необходимости нет. Поэтому актуально определение навыков, приобретаемых на тренажерах с учетом их адекватности.

Согласно классической схеме построения тренажеров [10] основным его элементом выступает моделирующее устройство, выполненное на базе ЭВМ (ПЭВМ) и состоящее из совокупности имитационных моделей. Методы теоретических исследований теории математического моделирования во многом опираются на достижения смежных наук: кибернетики, вычислительной техники, эргономики, имеющих некоторое специфическое преломление для практического использования в процессе создания моделей для тренажера РЭС. Так как любая имитационная модель - это лишь некоторый примерный прообраз реального объекта, то полученный с ее помощью результат необходимо проверять на предмет достоверности. К требованию достоверности результатов имитационного моделирования объектов исследования примыкает весьма важное требование - адекватность имитационной модели по отношению к объекту моделирования $[11,12]$. Использование имитационных моделей с ограниченной адекватностью в тренажере приводит к возникновению конфликта применения тренажеров, заключающегося в некорректном определении содержательного компонента учебных планов - количества тренировок на средствах тренажа.

Поэтому одной из актуальных научных задач является разработка метода формирования содержательного компонента учебных планов, защищенного от конфликта применения тренажеров, существующего из-за их ограниченной адекватности.

Для защиты содержательных компонентов от конфликта применения тренажеров в первую очередь необходимо определить индикатор сопутствующего признака этого конфликта. А именно нужно выработать понятие адекватности тренажеров в количественной форме и в формализованном виде определить влияние адекватности на требуемое количество тренировок.

\section{Заключение}

Достижение устойчивости АС управления ТП операторов к конфликтам применения РЭС и тренажеров на структурном уровне необходимо рассматривать как способность к целенаправ- 
ленному изменению структуры на основании оценки действий операторов, условий функционирования РЭС, степени адекватности и эксплуатационных ограничений тренажных средств. Конфликтоустойчивость как способность выполнять свои функции по предназначению в условиях возникновения конфликтов должна достигаться в АС управления ТП не только за счет селективных возможностей, обеспечивающих устранение ошибочных действий операторов, но и за счет выявления полезных содержательных компонентов учебных упражнений и учебных планов, определяющих учебные курсы. Поэтому в АС управления ТП совместно с традиционными методами выявления конфликта, вызванного отклонениями действий операторов от предписанных, целесообразно использовать так называемые индикаторы сопутствующих признаков, предназначенные для индикации конфликтов применения РЭС и тренажеров.

\section{Список литературы}

[1] Назаров Т.И., Потапов А.Н. Анализ системы организации тренажной подготовки операторов радиоэлектронных объектов управления воздушным движением, Труды XXIV межвузовской научно-технической конференции «Военная радиоэлектроника: опыт использования и проблемь, подготовка специилистов», С-Пб.: ВУНЦ ВМФ, 2013, 334-338 [Nazarov Т. I., Potapov A. N. Analysis of the system organization of training preparation of operators of radio-electronic facilities of air traffic control, Proceedings of the XXIV inter-University scientific and technical conference «Radio electronics Military: experience and problems of preparation of specialists», $\mathrm{S}-\mathrm{Pb}$.: The Russian Navy, 2013, 334-338 (in Russian)]

[2] Назаров Т.И., Потапов А.Н. Архитектура структурной конфигурации конфликтноустойчивой автоматизированной системы управления тренажной подготовкой операторов радиоэлектронных объектов, Труды II всероссийской научно-практической конференции «Академические Жуковские чтения», Воронеж: ВУНЦ ВВС «ВВА», 2014, 132-135 [Nazarov T. I., Potapov A. N. Architecture structural configuration konfliktno-sustainable automated control system of training the training of operators of electronic objects, Proceedings of II all-Russian scientificpractical conference «Academic Zhukovskie reading», Voronezh: VUNTS VVS «VVA», 2014, 132135 (in Russian)]

[3] Потапов А.Н. Особенности профессиональной подготовки операторов при использовании компьютерных систем тренажа, Сборник статей Всероссийской научно-технической конференции «XXXIV научные чтения, посвященные разработке творческого наследия К.Э. Циолковского», Калуга: Калужский дом знаний, 1999, 181-184 [[Potapov A. N. Features of professional training of operators in the use of computer systems training, Collection of articles all-Russian scientific-technical conference «XXXIV scientific readings, devoted to the development of the creative heritage of K. E. Tsiolkovskogo», Kaluga: Kaluga house of knowledge, 1999, 181-184 (in Russian)]

[4] Потапов А.Н., Дикарев В.А. Оценка качества программного обеспечения при разработке адаптивных тренажных систем подготовки лиц группы руководства полетами, Сборник статей научно-практической конференции «III Качинские чтения», Волгоград: Качинское ВВАУЛ,1998, 201-206 [Potapov A. N., Dikarev V. A. Assessment of software quality in the development of adaptive training systems training group flight management, Collected papers of scientific-practical conference «III Kaczynski readings», Volgograd: the Kachin VVAUL,1998, 201-206 (in Russian)] 
[5] Потапов А.Н. Автоматизация тренажной подготовки операторов радиоэлектронных объектов управления воздушным движением. Воронеж: ВАИУ, 2010, 136с. [Potapov A. N. Automation training training of operators of radio-electronic facilities of air traffic control. Voronezh: VAYU, 2010, 136 p. (in Russian)]

[6] Потапов А.Н. Методы и модели повышения эффективности эрготехнических компьютерных систем тренажа на основе оценки их конфликтно-устойчивости. Воронеж: ВАИУ, 2011, 96c. [Potapov A. N. Methods and models for increasing the efficiency of computer systems ergo technical training based on the assessment of their conflict-sustainability. Voronezh: VAIO, 2011, 96 p. (in Russian)]

[7] Губсков Ю.А., Иванова О.Г., Лебедев В.В., Овчаров В.В., Потапов А.Н., Черников В.В. Конфликтно-устойчивая система формирования информационного контента практической подготовки операторов эргатехнических систем. Приборы и системы. Управление, контроль, диагностика, М.: 2011, №8, 8-13 [Guskov Y. A., Ivanova O. G., Lebedev V. V., Ovcharov V. V., Potapov A. N., Chernikov V. V. Konfliktno-sustainable system of formation of the informational content of practical training for operators ergotamina systems. The devices and systems. Management, control, diagnostics, M.: 2011, No. 8, 8-13 (in Russian)

[8] Дикарев В.А., Гершкович И.С. Способ разрешения некоторых конфликтов, наблюдаемых при использовании компьютерных систем тренажа в профессиональной подготовке операторов, Сборник тезисов докладов Всеармейской научно-методической конференции «Проблемь внедрения новых информационных технологий в жизнедеятельность вуза», Тамбов: ТВАИИ, 1999, 77-80 [Dikarev V. A., Gershkovich, I. S. Method of resolving some of the conflicts observed in the use of computer systems training in the professional training of operators, Book of abstracts of the all-Army scientific-methodical conference «Problems of introduction of new information technologies in the activity of the University», Tambov: TVAII, 1999, 77-80 (in Russian)]

[9] Потапов А.Н., Сысоев Е.С. Особенности функционирования эргатических радиоэлектронных средств управления воздушным движением, Сборник статей региональной научнопрактической конференции курсантов, студентов, молодых ученых «Научные чтения имени A.C. Попова», Воронеж: ВУНЦ ВВС «ВВА», 2012, 38-42 [Potapov A. N., Sysoev E. S. Features of functioning of ergatic of electronic equipment for air traffic control, Collection of articles of regional scientifically-practical conference of students and young scientists «Scientific readings of a name of A. S. Popova», Voronezh: VUNTS VVS "VVA", 2012, 38-42 (in Russian)]

[10] Потапов А.Н. К вопросу разрешения конфликтов в практической подготовке лиц группы руководства полетами, Материалы I Всероссийской научно-технической конференции «Теория конфликта и ее приложение», Воронеж: ВГТА, 2000, 24-27 [[Potapov A. N. The issue of conflict resolution in the practical training the group flight management, Proceedings of the I allRussian scientific-technical conference «Conflict theory and its application», Voronezh: VGTA, 2000, 24-27 (in Russian)]

[11] Потапов А.Н., Назаров Т.И. Теоретические аспекты идентификации сопутствующего признака конфликта применения радиоэлектронных объектов. Научно-теоретический и практический журнал «Вестник Тамбовского Университета». Серия: Естественные и технические науки, 2012, Том 17,вып.6, 1603-1608 [Potapov A. N., Nazarov T. I. Theoretical aspects of the identification of concomitant symptom of the conflict-use of electronic objects. Scientific-theoretical 
and practical journal «Bulletin of Tambov University». Series: Natural and technical Sciences, 2012, Vol. 17,issue.6, 1603-1608 (in Russian)]

[12] Потапов А.Н. Моделирование информационных эргатических радиоэлектронных систем. Вестник Воронежского государственного технического университета, 2013, Том 9, №4, 22-25 [Potapov A. N. Modeling of ergatic information electronic systems. Bulletin of Voronezh state technical University, 2013, Vol. 9, No. 4, 22-25 (in Russian)] 